\title{
Plataforma Arduino como apoio ao ensino de programação no curso de Técnico em Informática integrado
}

\author{
Otávio Soares Paparidis, Matheus Eloy Franco
}

\author{
Área de Computação - Instituto Federal de Educação, Ciência e Tecnologia do Sul de \\ Minas Gerais - Campus Machado - 37750-000 - Machado - MG - Brasil \\ otaviopa@gmail.com, matheus.francolifsuldeminas.edu.br
}

\begin{abstract}
Resumo. Programação de computadores é uma das disciplinas essenciais e introdutórias em cursos de tecnologia da informação. Entretanto, muitos alunos enfrentam dificuldades nessa disciplina, causando altos índices de reprovação e evasão. Diante disso, a plataforma Arduino aparece como um meio de colocar a programação em prática. Algumas pesquisas já validaram essa proposta para estudantes de nível superior. Nesse cenário, este artigo propõe a utilização do Arduino para demonstrar conceitos de programação a alunos de nível técnico do IFSULDEMINAS. Os resultados obtidos se mostram satisfatórios e constatam a capacidade dessa abordagem prática para uma possível minimização das taxas de reprovação e evasão.
\end{abstract}

\begin{abstract}
Computer programming is one of the essential and introductory courses in information technology degrees. However, many students struggle in this discipline, resulting in high rates of failures and dropouts. Given this scenario, Arduino platform appears as a way to put into action basic programming concepts. Some researches already validated this proposal for students at undergraduate level. In such context, this paper proposes to use Arduino to demonstrate computer programming topics to secondary technical level students of IFSULDEMINAS. The results were satisfactory and attest the capability of this hands-on approach for a possible reduction in failure and dropout rates.
\end{abstract}

\section{Introdução}

Devido à vasta utilização de computadores nas tarefas do mundo atual, disciplinas de computação estão presentes nas matrizes curriculares dos mais variados cursos de todos os níveis de educação, do ensino fundamental à pós-graduação (VALENTE, 1999). Uma dessas disciplinas é a programação de computadores, marcada por dificuldades enfrentadas por educadores e educandos (Castro et al, 2003; Robins, Rountree, J. e Rountree, N., 2003; Delgado et al, 2004; Miliszewska e Tan, 2007; Macedo e Prietch, 2013).

Dentre as dificuldades notadas está a assimilação de conceitos básicos de algoritmos, que formam a base para a programação de computadores. Com a finalidade de melhorar a assimilação de conteúdos, os educadores recorrentemente utilizam objetos de aprendizagem, que podem ser definidos como uma coletânea de itens de conteúdo, prática e avaliação que são combinados com base em um único objetivo de aprendizagem (Gazzoni et al, 2006). 


\section{Ensino de Programação}

O ensino de computação é considerado como desafiador tanto para alunos de nível superior quanto para os do médio/técnico. Para Delgado et al (2004), as dificuldades "afloram principalmente no transcorrer das disciplinas ligadas ao estudo de algoritmos e programação e consideradas essenciais em todos os currículos de cursos de graduação em computação".

A complexidade dos conceitos estudados na computação é a razão pela qual Winslow (1996, apud Robins, Rountree, J. e Rountree, N. 2003), reitera que "uma boa pedagogia requer ao professor manter os fatos, modelos e regras simples no início, e somente expandi-los e melhorá-los à medida que os alunos ganham experiência". Como consequência das dificuldades enfrentadas, as disciplinas de programação enfrentam altas taxas de reprovação, que por sua vez afetam diretamente as taxas de evasão do curso (Castro et al, 2003; Robins, Rountree, J. e Rountree, N., 2003; Miliszewska e Tan, 2007; Macedo e Prietch, 2013).

Segundo Castro et al (2003), o modo como a programação é ensinada tem impactos negativos em cursos que possuem esta disciplina na matriz curricular dos primeiros anos. Ainda segundo os autores, esse impacto também atinge alunos que foram introduzidos à programação ainda no ensino médio.

Para Miliszewska e Tan (2007), outra dificuldade enfrentada pelos alunos “[...] é a necessidade de imaginar e compreender vários termos abstratos que não têm equivalentes na vida real: como uma variável, um tipo de dado, ou um endereço de memória são relacionados a um objeto da vida real?"

Diferentes estudos como o de Crouch et al (2004), miram formas de facilitar o ensino de disciplinas que exigem abstração. Uma das abordagens mais utilizadas pelos educadores é a utilização de demonstrações e exemplos práticos para facilitar a assimilação de conteúdos abstratos. Porém, segundo Crouch et al (2004), o simples uso de demonstrações e exemplos não é suficiente: alunos que observam passivamente demonstrações compreendem tanto quanto alunos que sequer as observam. Em contrapartida, a assimilação do conteúdo prospera quando o aluno se envolve com a demonstração.

Com o intuito de aprimorar esse processo de ensino, Robins, Rountree, J. e Rountree, N. (2003) reiteram a necessidade do uso de demonstrações em sala. Enfatizam também a importância de explicar cada passo do processo de criação do algoritmo a ser estudado. Desta maneira, observa-se que o ensino de programação se mostra como uma disciplina importante que apresenta dificuldades em seu processo de ensino aprendizagem; assim, novas abordagens podem ser utilizadas a fim de melhorar a exemplificação do conteúdo e estimular a motivação dos educandos.

\section{A Plataforma Arduino}

O cofundador da companhia Arduino, Massimo Banzi, descreve a missão do Arduino como plataforma de prototipagem para "encontrar uma forma simples e rápida de se criar protótipos da maneira mais barata possível." Arduino é uma plataforma de prototipagem de código aberto baseada em hardware e software de fácil utilização. Placas Arduino são capazes de ler entradas - a luz em um sensor, uma mensagem no Twitter - e transformá-las em uma saída - a ativação de um motor, o acendimento de 
um LED. Você pode dizer à placa o que fazer por meio do envio de um conjunto de instruções para o microcontrolador da placa (Banzi, 2009).

Outro fator que faz com que a plataforma Arduino seja versátil é a possibilidade de se acoplar shields à placa do Arduino, expandindo assim a sua funcionalidade. As shields podem trazer funções de rede (com ou sem fio), visores de LCD, câmeras, leitores de cartão, entre outros (Banzi, 2009).

Dessa forma, a plataforma em questão possui um vasto rol de aplicações, que vai tanto de simples protótipos eletrônicos a grandes automações industriais, ou de pequenos carrinhos de controle remoto à central de voo de veículos aéreos não tripulados (Arduino LLC, 2015).

\section{Arduino no Apoio ao Ensino}

Como foi observado, o ensino de programação é constantemente alvo de estudos que visam identificar a melhor forma de fazê-lo. A fim de auxiliar o processo de exemplificação do conteúdo, em consonância com os estudos de Crouch et al (2004) e Robins, Rountree, J. e Rountree, N. (2003), os professores lançam mão de equipamentos que não o computador. Dentre as possibilidades, figura a plataforma Arduino.

A simplicidade e a versatilidade, características do Arduino, fizeram com que este fosse objeto de estudos que visam utilizá-lo como ferramenta pedagógica. Segundo Macedo e Prietch (2013); Santos (2013) e Santos et al (2015), a plataforma Arduino faz com que os alunos se sintam mais motivados a aprender programação em comparação com aulas tradicionais que utilizam apenas o computador. Ainda de acordo com dois destes estudos, Prietch (2013) e Santos (2013), a assimilação do conteúdo das aulas é maior quando a plataforma Arduino está presente. Isso evidencia o envolvimento dos educandos com a programação entrando em consonância com a afirmação de Robins, Rountree, J. e Rountree, N. (2003) de que "precisamos motivar os estudantes, envolvêlos no processo, e fazê-los querer aprender a ser programadores eficazes".

Esse envolvimento dos educandos com a programação na prática vai ao encontro da teoria freiriana e de seus preceitos. Em sua obra, Freire (1987) salienta a importância da educação problematizadora, em contraponto ao que chama de "educação bancária". Segundo Freire, na educação bancária "não há criatividade, não há transformação, não há saber. Só existe saber na invenção, na reinvenção, na busca inquieta, impaciente, permanente, que os homens fazem no mundo, com o mundo e com os outros". Ainda segundo o autor, a criatividade é um elemento de papel preponderante na educação problematizadora e deve ser estimulada pelo educador-educando, de forma a potencializar o processo de ensino.

Segundo Narayan (2013), até os dias atuais o sistema de ensino sufoca a criatividade e o espírito inovador dos educandos, sugerindo a necessidade de uma mudança nos currículos escolares. Nesse contexto, o autor diz que a plataforma Arduino "pode desempenhar um papel fundamental no desenvolvimento cognitivo e criativo dos estudantes". Em seu estudo, Narayan combinou elementos sociais e tecnológicos a fim de potencializar o aprendizado dos estudantes. Essa combinação trouxe resultados positivos, visto que, além de estimular o ensino, os próprios alunos passaram a atuar como educadores e formadores de conteúdo, em consonância com a teoria freiriana. 
De acordo com a teoria de construção do conhecimento de Piaget (1967), o desenvolvimento cognitivo dos alunos é fruto da maturação biológica, em adição à interação com o meio, evidenciando a importância da experimentação no processo de aprendizagem. Tendo como base a teoria de Piaget, Papert (1980) cria uma extensão do construtivismo, denominado construcionismo. Ao definir o construcionismo, Papert (1980) afirma que a aquisição do conhecimento tem uma ligação muito forte com a interação com o objeto de estudo, seja refletindo sobre ele, seja agindo sobre o mesmo.

Correa (2012) afirma que Papert usou o termo construcionismo "para mostrar outro nível de construção do conhecimento, aquele que acontece quando o aluno constrói um objeto de seu interesse, como uma obra de arte, um relato de experiência ou um programa de computador". Ainda de acordo com Papert (1980, apud Correia, 2012) "o aprendiz constrói alguma coisa e o aprendizado só acontece quando o aprendiz constrói algo do seu interesse, porque o envolvimento afetivo torna a aprendizagem significativa".

Desse modo, dada uma padronização na utilização da plataforma Arduino para fins pedagógicos, um Objeto de Aprendizagem (OA) aflora. Segundo o Instituto de Engenheiros Elétricos e Eletrônicos (2000, apud Gazzoni et al, 2006) os OAs "[...] são definidos como uma entidade, digital ou não, que pode ser utilizada, reutilizada ou referenciada durante o processo de aprendizagem que utilize tecnologia". Para a elaboração deste OA, Gazzoni et al (2006) mostram a importância de levar em conta alguns aspectos, como a estrita ligação do OA com o conteúdo abordado em sala.

Seguindo esta mesma linha, trabalhos recentes como os de Cardoso e Antonello (2015) e Santos et al (2015) tiveram resultados positivos quanto à utilização do Arduino como OA. Entretanto, ambos analisaram o uso do Arduino por alunos de cursos superiores - no caso, bacharelados em Sistemas de Informação. Já o presente trabalho examina impactos deste OA sobre alunos de nível médio técnico.

\section{Materiais e Métodos}

Considerando o teor prático e os objetivos pedagógicos deste projeto, a metodologia do presente trabalho seguiu os pilares de pesquisa-ação: Investigação e Ação. Segundo Tripp (2005), a pesquisa-ação tem como ciclo básico as ações de: "Planejar uma melhora na prática, agir para implantar a melhora planejada, monitorar e descrever os efeitos da ação e avaliar os resultados da ação" (Tripp, 2005).

Assim, este trabalho visa alinhar os objetivos apresentados com as ações do ciclo supracitado. Inicialmente foi realizado um levantamento de demanda com três professores de disciplinas de programação de nossa instituição. Este levantamento foi realizado via entrevistas. O conteúdo da entrevista pode ser visto no formulário eletrônico via <http://goo.gl/forms/8UJul5Smu8>.

A partir do levantamento realizado com os professores, que culminou na confirmação unânime da demanda, deu-se início aos planos de elaboração dos projetos com Arduino a serem implementados nas aulas de programação dos alunos do IFSULDEMINAS - Campus Machado. Duas classes foram escolhidas para a implementação: turmas A e B do $1^{\circ}$ ano do curso integrado de Técnico em Informática. $\mathrm{O}$ critério para escolha foi o fato de ambas estarem passando pelo processo de introdução à programação, exatamente o público-alvo deste trabalho. A partir daí, foram 
estabelecidos três temas do ensino de programação a serem abordados: estrutura sequencial, estruturas condicionais e estruturas de repetição. Não por acaso, os temas escolhidos são a base da programação de computadores.

Para a criação dos modelos foi utilizada a ferramenta 123D Circuits, disponível no endereço <http://123d.circuits.io $>$. Trata-se de uma ferramenta web criada e mantida pela empresa Autodesk. Com ela, o usuário pode construir circuitos eletrônicos e simular o seu funcionamento. Além de ser gratuito, o simulador proporciona economia devido ao custo zero de investimento e manutenção. Como alguns alunos não têm familiaridade com eletrônica, é interessante que sempre simulem os projetos antes de montá-los efetivamente, para evitar a "queima" de placas e componentes. Isso deixa os alunos livres para trabalhar sua criatividade sem terem medo de provocar danos, e não abram mão da prática com os componentes reais, considerando que os projetos seriam replicados com os componentes reais nos casos das simulações bem-sucedidas.

Apesar de estar disponível apenas em inglês, o idioma não é um grande obstáculo para a utilização do simulador, pois sua interface é rica em recursos gráficos em oposição ao uso de textos, além de utilizar o formato "arrasta-e-solta" para a inclusão e manipulação dos componentes. A ferramenta ainda conta com mais duas opções de organização de tela: a utilizada para edição do código da placa Arduino (code editor) e a utilizada para a simulação propriamente dita, que pode ser iniciada pelo botão com um sinal de play e a inscrição "Start Simulation". A figura 1 representa a interface no modo de edição de código.

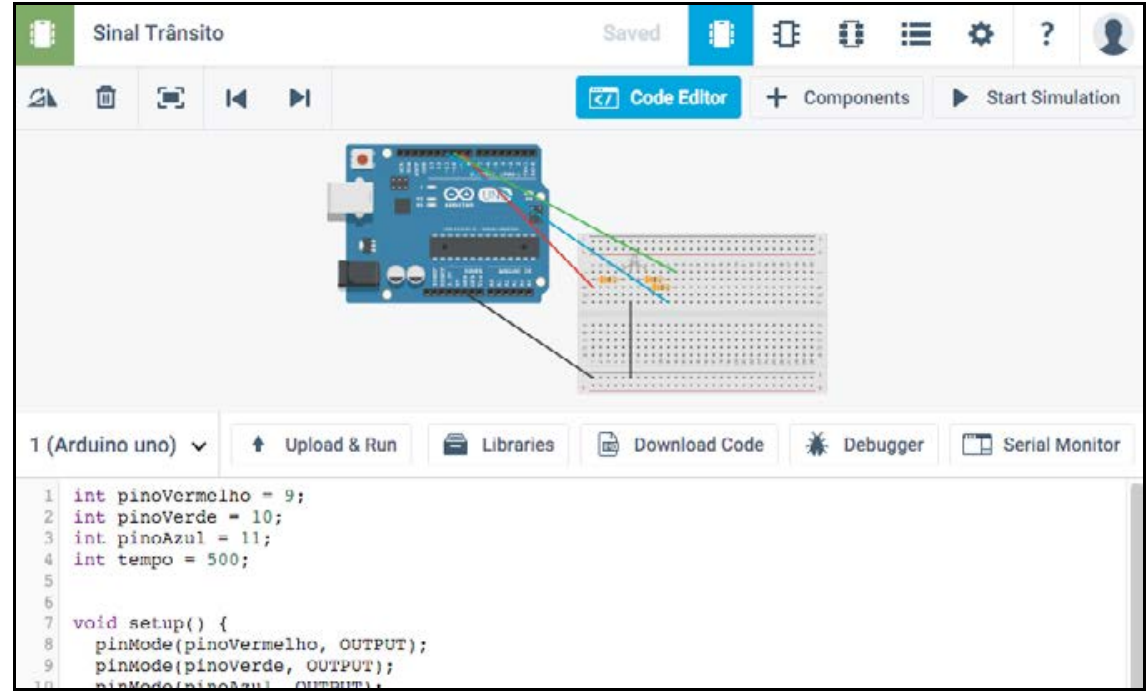

Figura 1: Interface do simulador 123D Circuits - Código.

Fonte: Autor (captura de tela)

Após a definição dos projetos e do simulador a ser utilizado, foram criados três projetos. Para exemplificar a estrutura sequencial, foi criado um simulador de semáforo. Para as estruturas condicionais, foi elaborado um simulador de poste de iluminação pública, que acende durante a noite e se apaga durante o dia. Por fim, para aulas sobre as estruturas de repetição foi utilizado um LED multicor que troca de cor repetidamente, mostrando um amplo espectro de cores. 


\section{Descrição dos Projetos Arduino}

Todos os projetos apresentados nesta seção encontram-se disponíveis publicamente nos seus respectivos endereços na plataforma 123D Circuits, por meio dos endereços: Projeto 1 - Estrutura Sequencial: <https://goo.gl/JdIcqS>, Projeto 2 - Estrutura Condicional: <https://goo.gl/QJVRaJ>, e Projeto 3 - Estrutura de Repetição: $<$ https://goo.gl/X3n6QT>. Nas páginas é possível visualizar a estrutura física, a lista de componentes utilizados, o código-fonte e a simulação do funcionamento do projeto.

Os modelos elaborados foram apresentados aos alunos. A demonstração visava deixar claro que a estrutura utilizada no algoritmo daquele projeto específico - por exemplo, a estrutura "Se-Então-Senão" - era exatamente a mesma que eles haviam aprendido ao estudá-la utilizando o método tradicional na tela do computador.

Como a estrutura física necessária para o funcionamento dos projetos 1 e 3 era a mesma, foi possível a utilização de apenas uma protoboard (ou placa de ensaio) para a demonstração de todos os três projetos, ficando uma montagem à esquerda e outra à direita. Cada projeto resultou em uma demonstração, identificada pelo mesmo número daquele. Para trocar de um projeto para outro bastava reconectar os fios na placa conforme etiquetas e fazer o carregamento do código correspondente ao projeto no Arduino. Todo o processo de troca leva cerca de um minuto, não atrapalhando, portanto, o andamento da aula. A figura 2 mostra a protoboard contendo as montagens supracitadas.

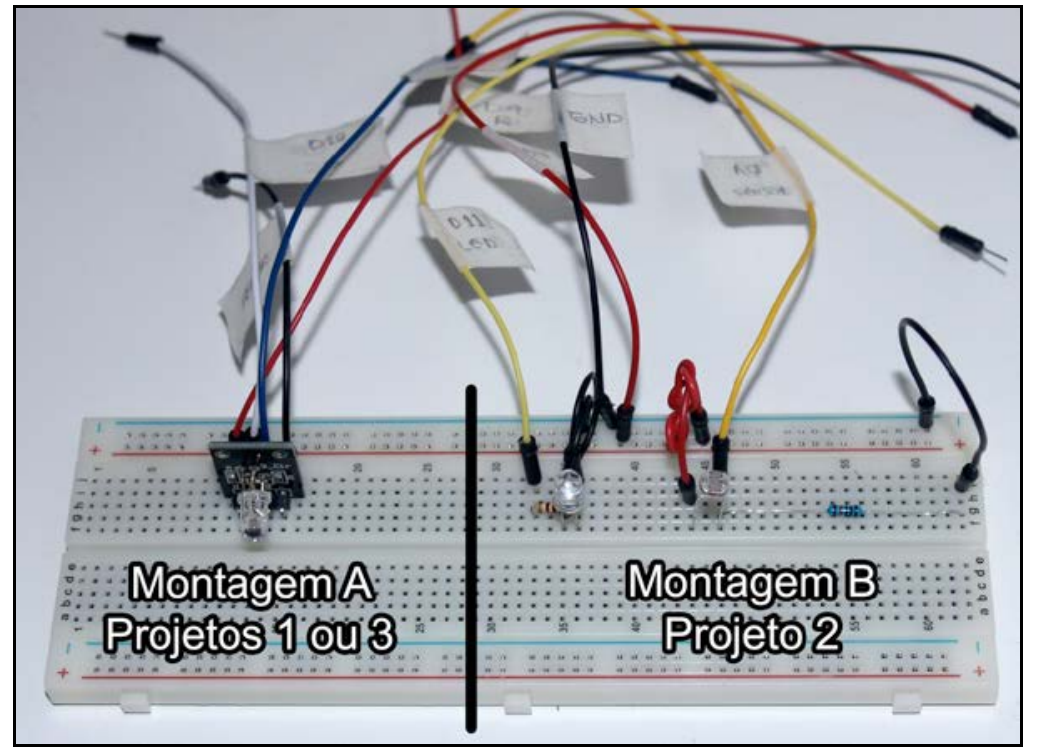

Figura 2: Protoboard com os projetos montados.

Fonte: Elaborada pelo autor.

\subsection{Demonstração 1 - Estrutura Sequencial}

A demonstração 1 tinha como objetivo explanar a estrutura sequencial de programação utilizando-se da sequência lógica de um semáforo. O código continha basicamente três pontos de execução: luz verde, luz amarela e finalmente a luz vermelha. Ao final, a própria estrutura de execução da plataforma Arduino se incumbia de repetir a sequência. 
Ao apresentar o funcionamento do algoritmo aos alunos, foram feitas algumas mudanças sutis no código a fim de atestar o papel desempenhado por cada trecho em específico: mudança no tempo de cada cor, inverter cores, pular uma das cores, etc. Dessa forma, os alunos puderam compreender que cada linha da sequência em questão produzia um efeito durante a execução do algoritmo.

\subsection{Demonstração 2 - Estrutura Condicional}

A demonstração 2, assim como na anterior, fazia uma analogia com um elemento que está presente no dia de todos que vivem em um ambiente urbano: um poste de iluminação. Assim como os postes da cidade, que acendem automaticamente quando está escuro e se apagam quando o sol nasce, o LED presente neste projeto acendia e apagava conforme a luminosidade ambiente.

A fim de reforçar o conceito da estrutura condicional, foram feitas várias mudanças no código, que tinha como base uma única estrutura "Se-Então-Senão". Salientou-se como uma simples mudança de um símbolo menor $(<)$ para maior $(>)$ invertia toda a lógica de funcionamento: o LED acendia quando a sala tinha as luzes acesas e se apagava quando estava com as luzes apagadas.

\subsection{Demonstração 3 - Estrutura de Repetição}

A terceira e última demonstração trazia um LED que mudava de cor rapidamente, exibindo um grande espectro de cores formadas pela manipulação das cores-fonte vermelho, verde e azul. Tal demonstração teve o propósito não só de exemplificar uma estrutura de repetição, mas também de mostrar como essas estruturas se comportam quando estão aninhadas dentro umas das outras. Para tal, o código trazia três estruturas "Para" (for) aninhadas de tal forma que cada uma delas controlava uma das cores-fonte.

Sendo definidos valores máximos para a intensidade de cada cor-fonte, o aluno pôde observar cada mudança destes valores e seu efeito sobre a formação da cor final, exibida pelo LED. Para facilitar essa percepção, durante a apresentação diminuiu-se o tempo de mudança entre as cores, além de exibir a intensidade de cada cor-fonte em tempo real na tela do computador, projetada para toda a sala.

\section{Avaliação e Resultados}

A proposta pedagógica foi avaliada por um total de 71 alunos. Para essa avaliação, foi aplicado um questionário semiestruturado, disponível em <http://goo.gl/forms/ 8UJul5Smu8>, que teve como referência o modelo de avaliação de jogos educacionais proposto por Savi et al (2010). Tal modelo, conforme relatado pelos autores, segue os "princípios da taxonomia de Bloom para avaliação do impacto na aprendizagem do aluno", sendo a escala Likert de 5 pontos utilizada para análise dos dados. O quadro a seguir mostra as questões utilizadas no formulário e seus respectivos aspectos avaliados, divididos em três grandes grupos: motivação, experiência e conhecimento. 
Quadro 1. Aspectos avaliados pelo questionário.

\begin{tabular}{|c|c|c|}
\hline \multirow{4}{*}{ Motivação } & Atenção & $\begin{array}{l}\text { Houve algo interessante no início das demonstrações com o Arduino } \\
\text { que capturou minha atenção. }\end{array}$ \\
\hline & Relevância & $\begin{array}{l}\text { Ficou claro para mim como o conteúdo das demonstrações com } \\
\text { Arduino está relacionado com coisas que eu já sabia. }\end{array}$ \\
\hline & Confiança & A quantidade de informações foi adequada aos exemplos. \\
\hline & Satisfação & $\begin{array}{l}\text { Eu aprendi algumas coisas com os exemplos que foram } \\
\text { surpreendentes ou inesperadas. }\end{array}$ \\
\hline \multirow[b]{2}{*}{ Experiência } & Imersão & Senti-me estimulado a aprender com o Arduino. \\
\hline & Desafio & $\begin{array}{l}\text { Eu gostei de aprender com o Arduino e não me senti ansioso ou } \\
\text { entediado. }\end{array}$ \\
\hline \multirow{2}{*}{ Conhecimento } & Divertimento & $\begin{array}{l}\text { Gostaria de ver mais demonstrações com Arduino para } \\
\text { complementar meu aprendizado em programação. }\end{array}$ \\
\hline & Compreensão & $\begin{array}{l}\text { Depois de ver exemplos com o Arduino consigo compreender e } \\
\text { aplicar melhor os temas apresentados nos exemplos. }\end{array}$ \\
\hline
\end{tabular}

Como proposto, ao final das apresentações nas salas de aula, os alunos foram convidados a responder o questionário; cerca de $80 \%$ dos alunos o fizeram. O interesse demonstrado pelos alunos em utilizar o Arduino para criar seus próprios experimentos foi nítido. Uma das preocupações iniciais do presente trabalho foi que, embora o Arduino seja uma plataforma de simples programação, talvez fosse visto como algo complicado para os alunos do nível técnico. Entretanto, não foram apresentadas dificuldades quanto a isso. Um dos alunos ainda vinculou a simplicidade do Arduino à compreensibilidade do mesmo: "Como demonstrado, o Arduino é bem fácil de aprender e aplicar, ou seja, bem fácil de se compreender". Outro destacou o estímulo proporcionado pelo Arduino como algo positivo: "Arduino é capaz de estimular a conhecer ainda mais a programação". As opiniões vão ao encontro das observações de Prietch (2013) e Santos (2013) no tocante ao papel do Arduino como facilitador da compreensão do conteúdo.

Ademais, os resultados obtidos nos segundo e terceiro eixos (experiência e conhecimento) evidenciaram o envolvimento dos alunos com o OA, além do estímulo à criatividade dos mesmos, fatores preponderantes para um ensino eficaz (Narayan, 2013; Freire, 1987). A figura 3 mostra um gráfico do tipo radar apresentando os resultados de todos os aspectos avaliados conforme o quadro 1.

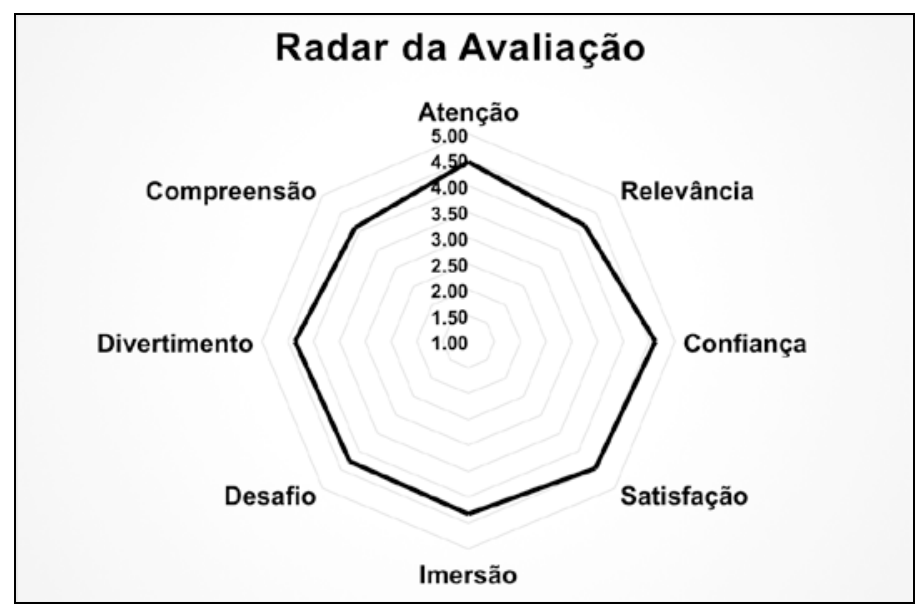

Figura 3: Gráfico Radar das Avaliações.

Fonte: Elaborada pelo autor. 


\section{Considerações Finais}

Diante dos dados obtidos, constatou-se que a abordagem proposta tem, de fato, capacidade de potencializar o ensino de programação não somente no ensino superior, mas também no ensino técnico integrado. $\mathrm{O}$ fator motivacional manifestado pelos alunos foi preponderante dentre os aspectos que confirmam essa capacidade. Além de se sentirem motivados, os educandos mostraram ter uma oportunidade de externar sua criatividade com a plataforma Arduino, seja em trabalhos propostos pelo professor, seja em projetos independentes.

O trabalho deixa um legado que poderá ser analisado e, eventualmente, incorporado a planos de ensino de qualquer curso que tenha em sua matriz curricular disciplinas de programação de computadores e tenha uma realidade similar às encontradas nos cursos de nossa instituição.

Como possíveis trabalhos futuros, sugere-se a utilização contínua da abordagem experimentada neste trabalho, fazendo com que os alunos tenham contato com a prática da programação com Arduino do início ao fim da disciplina, e não apenas pontualmente como foi neste estudo.

\section{Referências}

Arduino LLC. Arduino Forum. Arduino Website, 2015. Disponivel em: https://forum.arduino.cc. Acesso: Dezembro 2015.

Banzi, M. Getting Started with Arduino. [S.1.]: O'Reilly Media, 2009.

Cardoso, R. e Antonello, S. L. Interdisciplinaridade, programação visual e robótica educacional: relato de experiência sobre o ensino inicial de programação. Workshops do IV Congresso Brasileiro de Informática na Educação (CBIE). Maceió, 2015.

Castro, T., Castro, A., Menezes, C. Boeres, M. e Rauber, M. Utilizando Programação Funcional em Disciplinas Introdutórias de Computação. XI Workshop de Educação em Computação - WEI. Campinas, 2003.

Correa, M. D. C. Tecnologia e Práticas Educativas: O Projeto Mundo do Saber. Americana, 2012.

Crouch, C., Fagen, A. P., Callan, J. P. e Mazur, E. Classroom demonstrations: Learning tools or entertainment? American Journal of Physics, 72, 2004. 835-838.

Delgado, C., Xexeo, J. A. M., Souza, I. F., Campos, M. e Rapkiewicz, C. E. Uma Abordagem Pedagógica para a Iniciação ao Estudo de Algoritmos. XII Workshop de Educação em Computação - WEI. Salvador, 2004.

Freire, P. Pedagogia do oprimido. 17 a . ed. Rio de Janeiro, 1987.

Gazzoni, A., Canal, A. P, Falkembach, G. A. M., Fioreze, L. A., Pincolini, L. B. e Antoniazzi, R. Proporcionalidade e semelhança: Aprendizagem via objetos de aprendizagem. Revista Novas Tecnologias na Educação. Porto Alegre, 2006.

IEEE Standards Association. IEEE Learning Technology Standards Committee. IEEE.org, 2000. Disponível em: https://ieee-sa.imeetcentral.com/ltsc. 
Macedo, R. S. D. e Prietch, S. S. Utilizando a Placa Arduino como Ferramenta de Apoio ao Ensino de Programação. Anais da IV Escola Regional de Informática da SBC - Regional de Mato Grosso. 37-42. Alto Araguaia, 2013.

Miliszewska, I. e Tan, G. Befriending Computer Programming: A Proposed Approach to Teaching Introductory Programming. Issues in Informing Science and Information Technology. Melbourne, 2007.

Narayan, V. Technology as a creative partner: Unlocking learner potential and learning. 30th ascilite Conference. 612-621. Sydney, 2013.

Papert, S. Mindstorms. Children, Computers and Powerful Ideas. New York, 1980.

Piaget, J. e Inhelde, B. The Child's Conception of Space. New York: W. W. Norton \& Company, 1967.

Robins, A., Rountree, J. e Rountree, N. Learning and teaching programming: a review and discussion. Computer Science Education, 13, 2003. 137-172.

Santos, A., Gorgônio, A., Lucena, A. e Gorgônio, F. A Importância do Fator Motivacional no Processo Ensino-Aprendizagem de Algoritmos e Lógica de Programação para Alunos Repetentes. XXIII Workshop sobre Educação em Informática - WEI. Recife, 2015.

Santos, R. R. D. Utilização de Plataformas de Hardware Aberto no Ensino: Um Estudo de Caso na Disciplina de Introdução à Engenharia de Computação. International Journal of Computer Architecture Education (IJCAE), v. 2, p. 37-40, 2013.

Savi, R., Wangenheim, C. G., Ulbricht, V., Vanzin, T. Proposta de um Modelo de Avaliação de Jogos Educacionais. RENOTE - Revista Novas Tecnologias na Educação, v. 8, n. 3. Porto Alegre, 2010.

Tripp, D. Pesquisa-ação: uma introdução metodológica. Educação e Pesquisa, v. 31, 443-466. São Paulo, 2005.

Valente, J. A. O computador na sociedade do conhecimento. UNICAMP/NIED. Campinas, 1999.

Winslow, L. E. Programming pedagogy - a psychological overview. SIGCSE Bulletin, 28, n. 3. 17-22. New York, 1996. 